\title{
Functional MRI reveals memories of mother in
}

\section{newborn chicks}

Mehdi Behroozi ${ }^{1,5^{*}}$, Elena Lorenzi $i^{2,5^{*}}$, Sepideh Tabrik ${ }^{3}$, Martin Tegenthoff ${ }^{\beta}$, Alessandro

4 Gozzi $^{4}$, Onur Güntürkün ${ }^{1}$, Giorgio Vallortigara ${ }^{2}$

6 Ruhr University Bochum, Universitätsstraße 150, 44780, Bochum, Germany

${ }^{2}$ Center for Mind/Brain Sciences, University of Trento, Piazza Manifattura 1, 38068

8 Rovereto (TN), Italy

${ }^{3}$ Department of Neurology, BG-University Hospital Bergmannsheil, Ruhr-University

10 Bochum, Bürkle-de-la-Camp-Platz 1, 44789, Bochum, Germany

$11 \quad{ }^{4}$ Functional neuroimaging laboratory, Istituto Italiano di Tecnologia, Rovereto, Italy

$12{ }^{5}$ These authors contributed equally to this work.

13 "Corresponding authors: mehdi.behroozi@ruhr-uni-bochum.de; elena.lorenzi@unitn.it; 


\section{$14 \quad$ Abstract}

15 Filial imprinting has been used as a powerful ethological paradigm to investigate the

16 neurobiology of early learning that affects lifelong behaviours. When a visually naïve chick

17 is exposed to one of a wide range of conspicuous objects, it may learn its characteristics

18 and subsequently recognises and selectively approaches this stimulus (usually the

19 mother hen and siblings). While the initial phases of memory acquisition have been

20 unravelled, the long-term storage and retrieval components of imprinting memories are

21 still unknown. Here, we used functional MRI in awake newly hatched chicks to identify the

22 long-term storage of imprinting memories in a wide network comprising the hippocampal

23 formation, the medial striatum, the arcopallium, and the prefrontal-like nidopallium

24 caudolaterale. These results provide the first example of non-invasive imaging of the brain

25 in a newborn vertebrate.

26

27 Keywords: fMRI; imprinting; brain; learning; memory acquisition; retrieval. 


\section{Introduction}

Filial imprinting is a learning process by which the young of some organisms can

30 learn about a conspicuous object, usually the mother or siblings, by simply being exposed

31 to it for a short period of time soon after birth (McCabe, 2013). It owes its great popularity

32 to the work of Nobel-prize-winning ethologist Konrad Lorenz (Lorenz, 1935), but it was

33 originally described by Douglas Spalding (Spalding, 1954) in the offspring of some

34 nidifugous (precocial) bird species, such as chicks or ducklings (see Vallortigara, 2021).

35 Visual imprinting has been mostly studied, though acoustic or olfactory imprinting can be

36 observed as well, the latter being prominent in mammals (Müller-Schwarze and Müller-

37 Schwarze, 1971).

$38 \quad$ Although in principle visual imprinting can occur with any kind of object, research

39 has shown that the process is actually assisted by a set of biological predispositions which

40 guide an animal's attention towards those object features that are more likely to be

41 observed in social partners - e.g., preferences in domestic chicks include simple features

42 such red colour (which is prominently observed in the head region of conspecifics), or

43 self-propelled motion (which is typical of living things), as well as more complex assembly

44 of features such as face-like stimuli or biological motion in point-light displays (review in

45 Lorenzi and Vallortigara, 2021; Vallortigara, 2021). Brain research has shown that

46 biological predispositions are associated with activation of areas of the so-called Social

47 Behavior Network, and in particular of the lateral septum for motion stimuli and of the

48 nucleus taenia (homologous of the mammalian medial amygdala (Yamamoto et al., 49 2005)) for face-like stimuli (review in Lorenzi and Vallortigara, 2021). 
Interest for filial imprinting quickly spanned from behavioural biology to psychological

51 development and psychopathology, inspiring for instance John Bowlby's theory of

52 attachment, which postulates a crucial role of the mother-child bond for subsequent

53 psychological development and, complementarily, the psychiatric outcomes associated

54 with early mother deprivation (recent reviews in Lemche, 2020; Vicedo, 2013).

55 In the 70's filial imprinting served as a model for the neurobiological investigation of

56 memory. Gabriel Horn and colleagues (review in Horn, 2004) identified an associative

57 brain region involved in the formation of an imprinting memory, the intermediate medial

58 mesopallium (IMM according to the new avian brain nomenclature; previously referred to

59 as IMHV, intermediate medial hyperstriatum ventrale (Jarvis et al., 2005; Reiner et al.,

60 2004)). IMM proved to be crucial during the acquisition phase of the visual imprinting

61 memory. More precisely, it was found that exposure to the imprinting object was

62 associated with changes in the left but not in the right IMM (Bradley et al., 1981; Horn et

63 al., 1985). Subsequent studies with auditory imprinting revealed that the imprinting-

64 related area extended ventrally into a medialmost nidopallial area, the nidopallium medial

65 pars medialis (NMm) (Bredenkötter and Braun, 2000, 1997). Here we will use the label

66 medial nidopallium/mesopallium (MNM) to jointly label the mesopallial and nidopallial

67 entity of the imprinting area.

68 Experiments involving sequential lesions, first to one side of IMM and subsequently

69 to the other (Cipolla-Neto et al., 1982; Horn et al., 1983), suggested that the store in the

70 left IMM is only temporary, and the right IMM is implicated in transferring information from

71 the left IMM to another, unknown brain region dubbed S', and that this transfer appears

72 to be complete within $6 \mathrm{~h}$ after the end of exposure (Davey et al., 1987). Thus, to cite 
73 Gabriel Horn's words “We are still some way from being able to visualize, through the

74 microscope or by using brain imaging techniques, the neural trace of (imprinting) memory"

75 (Horn, 2004). Here we set out to find an answer to this open question.

76 Techniques such as functional magnetic resonance imaging (fMRI) would allow for

77 non-invasive investigation of ongoing brain activity and look ideally-suited for the time-

78 course of the formation of an imprinting memory. Here we therefore devised an fMRI

79 study in awake newly-hatched chicks to identify the imprinting network and the long-term

80 store of imprinting memories.

81 We exposed (imprinted) chicks on either a preferred (red) or a non-preferred (blue)

82 colour (Maekawa et al., 2006; Salzen et al., 1971). After exposure, chicks were tested

83 with a sequence alternating the two colours in the scanner. Chicks imprinted on red colour

84 showed activity in pallial and subpallial brain regions involved with storage and memory

85 retrieval, such as the medial striatum, the arcopallium, the hippocampus, and the

86 nidopallium caudolaterale (a presumed avian equivalent of mammalian prefrontal cortex).

87 Chicks imprinted on blue showed little or no activity in the same regions but, as a result

88 of exposure in the scanner to the preferred red colour, started a process of secondary

89 imprinting, which confirmed an early activation of mesopallium, as well as a precocial

90 involvement of the Social Behavior Network during the first exposure to a predisposed

91 feature, such as the colour red. We thus for the first time were able to image both the

92 brain areas involved in the acquisition of the imprinting memory, with associated areas

93 involved in biological predispositions that canalise the learning process, and the areas -

94 unknown up to now - associated with long-term store of imprinting memory.

\section{Results}




\section{Establishment of a fully non-invasive and awake fMRI for}

\section{7 the chicks}

98 To enable whole-brain fMRI acquisition in awake chicks, we developed a fully non-

99 invasive set-up to minimise head and body movements (Fig. 1B). Before fMRI scans,

100 chicks were imprinted for two days on either a preferred red or a non-preferred blue light

101 ball (Salzen et al., 1971). At first, we tried to habituate newborn chicks to the head fixation

102 system before the scanning session. However, the habituation sessions stressed the

103 animals so much that ended up panicking against the head fixation system already at the

104 second habituation session. Therefore, we did not habituate the animals to the head

105 fixation before the scan, in order to avoid any association between stress or anxiety and

106 the head fixation system. So before scanning, chicks were only habituated to the scanner

107 noise (Fig. 1A). On the third day, after wrapping the animal in a paper tissue to avoid any

108 body-part movements (such as wings and legs), blocks of plasteline were used to

109 comfortably fixate the head, minimising movements and scanner's noise by covering their

110 ears (Fig. 1B).

111 To record the spontaneous resting-state and task-based BOLD signals, a single-

112 shot multi-slice rapid acquisition with relaxation enhancement (RARE) sequence was

113 adopted from Behroozi et al (Behroozi et al., 2020, 2017). Voxel-wise signal-to-noise ratio

114 (SNR) and temporal SNR (tSNR) were calculated over the resting-state (rs-fMRI) and

115 task-based fMRI (tb-fMRI) scans respectively. The tSNR of the RARE sequence in each

116 voxel was calculated after applying motion correction and high-pass temporal filtering

117 (cut-off at 120s) to remove any linear drift. Temporal SNR in the entire telencephalon 
118 ranged from 50 to 100 (Fig. S1A, B) for both tb- and rs-fMRI scans. Furthermore, the

119 result indicated highly correlated SNR and tSNR for both rs- and tb-fMRI scans (Fig. S1C,

120 D).

A
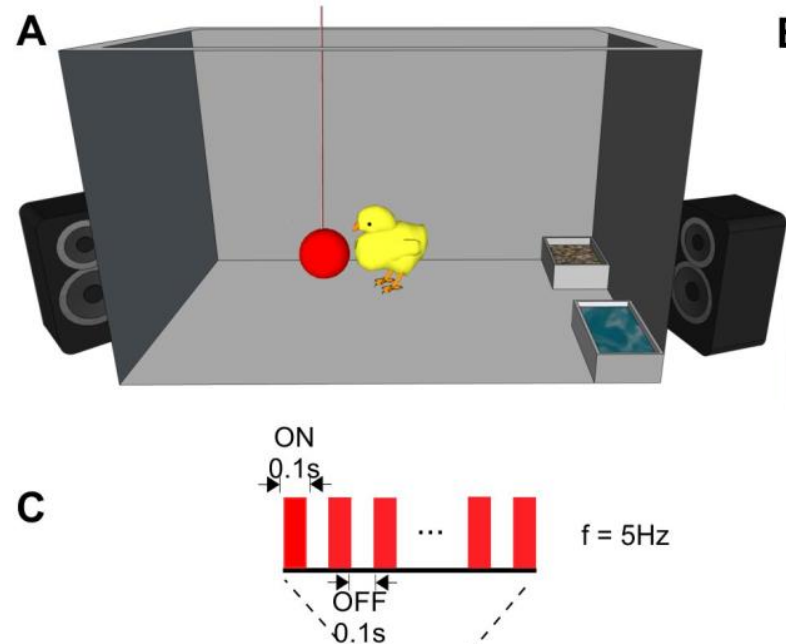

B

B
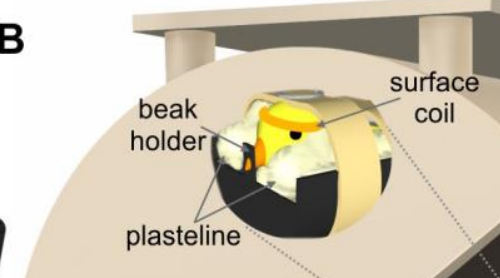

$+2$

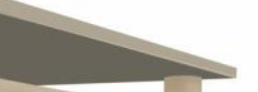

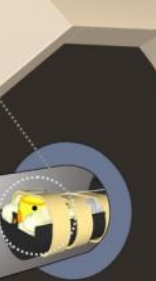

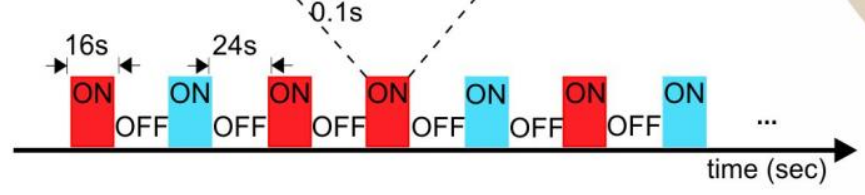

Fig.1- Experimental setups and stimulation sequence for awake chick fMRI. (A) Imprinting cage. Newborn chicks were first exposed to a hollow plastic ball with a flickering red/blue light at a frequency of $5 \mathrm{~Hz}$. (B) Custom-made restrainer and 7T fMRI system. Awake chicks were placed in an MR-compatible tube. To immobilise non-invasively the animals, a beak holder was used to control the beak movements, and blocks of plastelines were used to cover the ears and reduce head movements. To avoid body-part movements, animals were wrapped in paper tissue before fixating the head. Subsequently, the animal's body was taped to the restrainer. (C) A sequence of the block design experiment paradigm. Visual stimuli were presented in blocks of $16 \mathrm{~s}$ followed by $24 \mathrm{~s}$ dark. During the ON blocks, the visual stimulus (red/blue light) flickered at a frequency of $5 \mathrm{~Hz}$.

In order to verify that adequate fixation was achieved during fMRI scans, we used

133 the realignment parameters and the results of the frame-wise displacement (FD) to

134 evaluate the amount of head motion. Overall, the custom-made restrainer yielded a low

135 level of head movements. There was only $2.02 \%$ ( 218 volume) and 1,08\% (19 volumes)

136 of fMRI volumes with FD higher than $0.2 \mathrm{~mm}(\sim 40 \%$ of voxel size) over all subjects in the 
137 task-based and resting-state experiments, respectively (Figure 2A). The median of frame-

138 wise displacement was $\sim 0.03 \mathrm{~mm}$ for both tb-fMRI and rs-fMRI experiments. However,

139 most head movements occurred in the $y$-direction (Figure 2B, C). The respective violin

140 plot information for translations in $y$-direction is as follow: tb-fMRI: $\max / \min =0.22 /-0.31$

141 and median $\sim 0$; and $\mathrm{rs}-\mathrm{fMRI}: \mathrm{max} / \mathrm{min}=0.28 /-0.30$ and median $\sim 0$. The higher motion

142 parameters in the y-direction were most likely due to the design of the head restrainer,

143 which allowed movements in the dorsoventral direction to avoid blocking the throat.
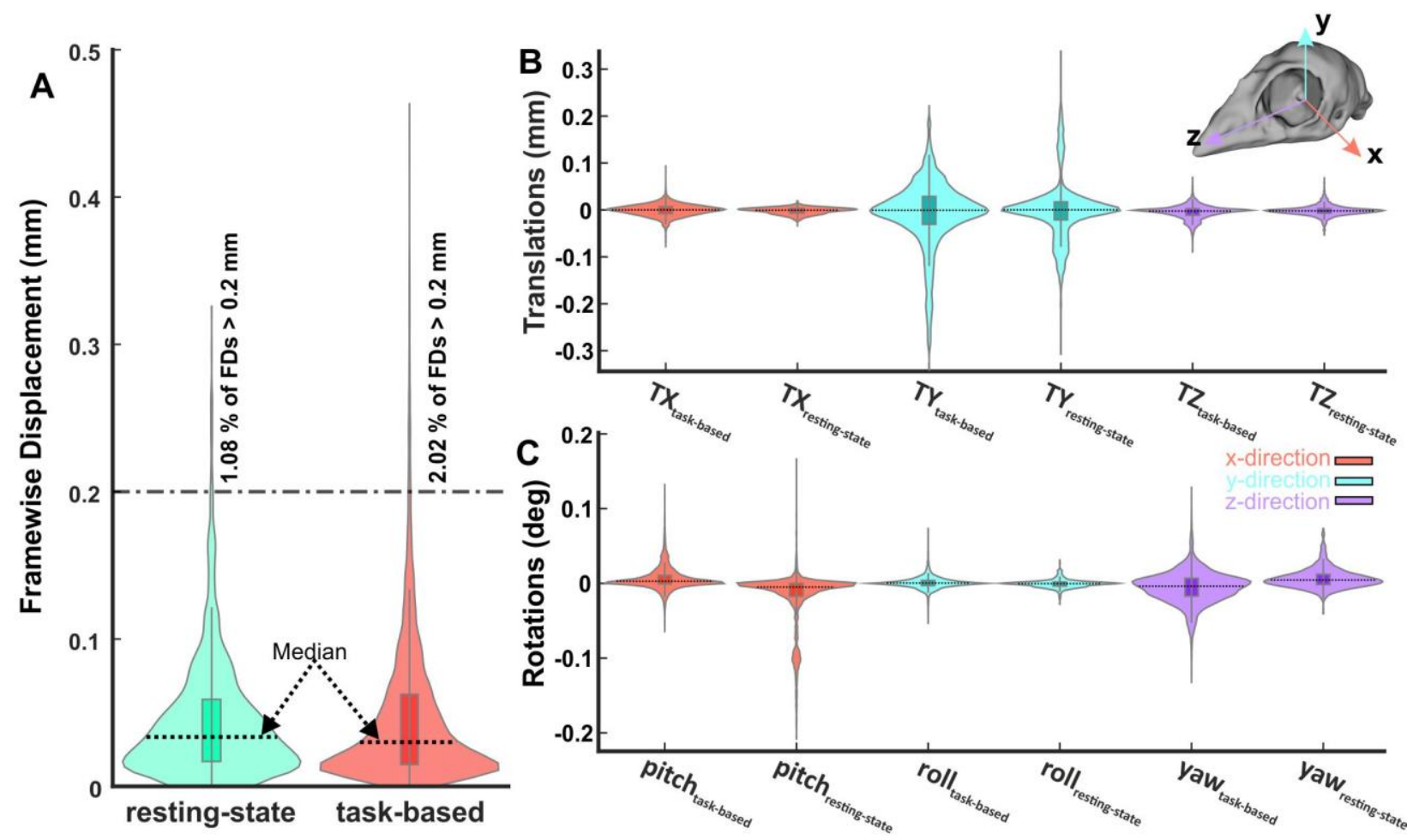

145 Fig.2- Characterisation of the head movements. (A) Violin plot of the frame-wise displacement 146 over all subjects for task-based $(n=17)$ and resting-state $(n=9)$ fMRI scans, respectively. The 147 median is represented by a dash-line across the kernel density estimate of the data. $(B, C)$ Violin 148 plot of estimated motion parameters. Motion parameters were estimated by a $3 \mathrm{D}$ rigid body model 149 with six degrees of freedom for translation ( $x, y$, and $z$-direction) and rotation (pitch, roll, and yaw). 150 The median is represented by a dash-line across the kernel density estimate of the data. 


\section{Distinct BOLD response to identify the acquisition and}

\section{3 long-term storage of imprinting memory}

154 We recorded the whole brain BOLD signals from 17 head-restrained awake chicks

155 already imprinted to either a preferred colour, red $(n=9)$, or a non-preferred colour

156 (Maekawa et al., 2006; Salzen et al., 1971), blue $(n=8)$. During fMRI scanning, animals

157 were presented with both colours (Fig. 1C), which depending on the previous imprinting

158 training could represent either the imprinted or the control colour. The two colours were

159 presented in a block design manner and a pseudo-random order (48 trials, 24 per

160 condition, see Methods). For the preferred colour group, the imprinting colour ( $\operatorname{Imp}$ ) was

161 red and the control (Cont) was blue, while for the non-preferred colour group the

162 imprinting colour was blue and the control red. To identify the long-term storage of

163 imprinting memory, we used the contrast of $\operatorname{Imp}>$ Cont in a conventional generalised

164 linear model (GLM) based statistical analysis. The first-level results at the single-subject

165 level were then entered into a second-level analysis (random-effect modelling, $Z=3.1$

166 (red group) and $p<0.05$ family-wise error $(F W E)$ ) to illustrate the activation clusters at

167 different networks of chick prosencephalon.

168 Before $\mathrm{MRI}$ scans, chicks were exposed to the imprinting stimulus for 2 days, during 169 which they learned the feature of the imprinting object and stored them as a long-term 170 memory (McCabe, 2013). Therefore, we expected to find activation in regions involved in

171 memory retrieval. While $\mathrm{Imp}>$ Cont contrast in the red group showed robust activation

172 clusters in many telencephalic as well as diencephalic regions, $\operatorname{Imp}>$ Cont contrast in the

173 blue group showed no significant activation clusters. 
174 This is due to chicks' preference for red over blue (Maekawa et al., 2006), therefore

175 we used the Cont > Imp contrast (red > blue colour) during the last 20 minutes of

176 scanning, to investigate the memory formation phase of a secondary imprinting process

177 (Boakes and Panter, 1985) elicited by the presence of the preferred colour red. As

178 illustrated in Figure 3, the voxel-based group analysis showed robust BOLD responses in

179 different visual prosencephalic regions: the nucleus geniculatus lateralis pars dorsalis

180 (GLd, which receives direct input from the retina (Güntürkün and Karten, 1991)), the right

181 intermediate hyperpallium apicale (IHA, which primarily receives visual thalamic input

182 (Csillag and Montagnese, 2005)), the right hyperpallium intercalatum (HI) and right

183 hyperpallium densocellulare (HD), and bilaterally the hyperpallium apicale (HA, together

184 with HD associative hubs of the thalamofugal pathway (Csillag and Montagnese, 2005;

185 Shanahan et al., 2013)) of the thalamofugal pathway, bilaterally the nucleus rotundus

186 (Rot, which is the primary thalamic input region of the tectofugal pathway). Also parts of

187 the auditory system were activated: bilaterally the ventromedial part of the Field-L

188 complex and the right nucleus ovoidalis (OV), a thalamic auditory nucleus receiving direct

189 input from the avian homologue of the inferior colliculus (torus semicircularis (Karten,

190 1967)) that projects to Field-L. We detected significant activation clusters in the

191 associative pallial regions nidopallium medial pars medialis (NMm) and bilaterally in the

192 caudal intermediate medial mesopallium (IMM). Within the two interconnected Social

193 Behavior Network and Mesolimbic Reward System, we detected a significant BOLD

194 increase rightward in the bed nucleus of the stria terminalis (BNST), the nucleus

195 accumbens (Ac) and the medial striatum (MSt), bilaterally in the septum and leftward in

196 the posterior pallial amygdala (PoA) and the ventromedial part of hippocampus (Hp-VM). 

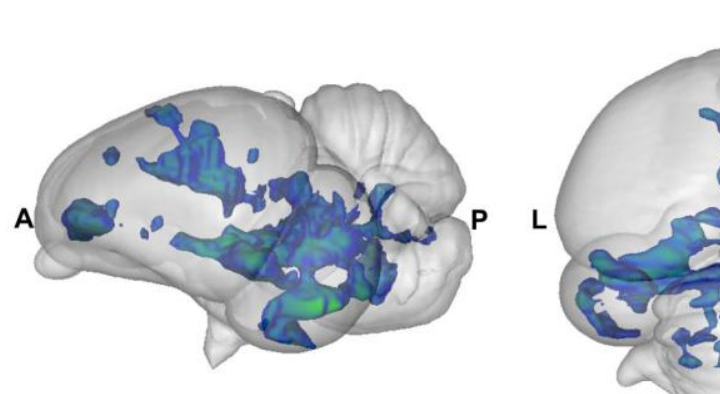

A
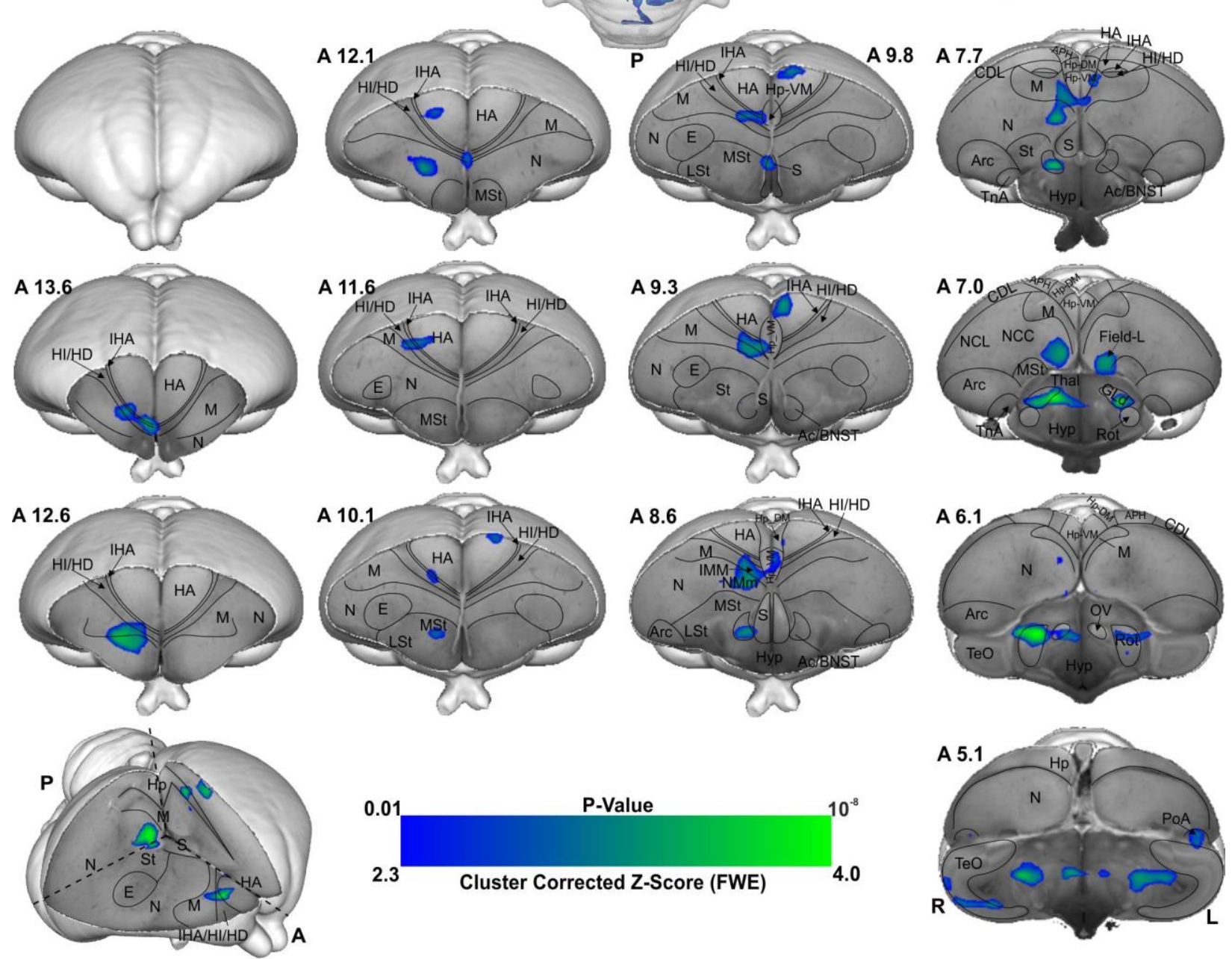

Fig.3- BOLD response pattern during the acquisition of imprinting memory. Statistical maps for the BOLD signal increase in the contrast of red light versus blue light in the Blue group $(n=8, Z$ $=2.3$, and $p<0.05$ FEW corrected at the cluster level). The top row images show the 3D representation of the activation pattern inside a translucent chick brain. A 3D depiction of the chick brain is represented at the bottom of the left column with an example window at the level of A 7.0. Anatomical borders (black lines) are based on the contrast difference of ex-vivo chick brain and Chick atlas (Herold et al., 2014; Kuenzel and Masson, 1988). The corresponding abbreviations of ROls are listed in the Table S1. 
As illustrated in Figure 4, the voxel-based group analysis during the imprinting 208 memory retrieval phase in the red group showed robust BOLD responses in different 209 visual prosencephalic regions: the right GLd, bilaterally in IHA, HI, HD and HA (Fig. 4). 210 We found also a significant BOLD rightward increase in part of the auditory system, OV. 211 Furthermore, we detected a significant increase in the BOLD signal in the 212 associative right MNM (IMM + NMm) and nidopallium caudolaterale (NCL) and in left 213 portions of the caudal mesopallium dorsale (MD) and nidopallium caudocentrale (NCC;

214 all interconnected regions (Aoki et al., 2015; Atoji and Wild, 2009; von Eugen et al., 215 2020)).

216 Within the two interconnected Social Behavior Network and Mesolimbic Reward 217 System, we detected significant bilateral activation in the ventromedial part of the 218 hippocampus (Hp-VM), while rightward activation clusters in the bed nucleus of the stria 219 terminalis (BNST), in the nucleus accumbens (Ac), in the medial striatum (MSt), in the 220 medial and dorsal arcopallium (respectively AM and AD), in the posterior pallial amygdala 221 (PoA) and in the preoptic, anterior and ventromedial areas of the hypothalamus 222 (respectively POA, $\mathrm{AH}$, and $\mathrm{VMH}$ ). 


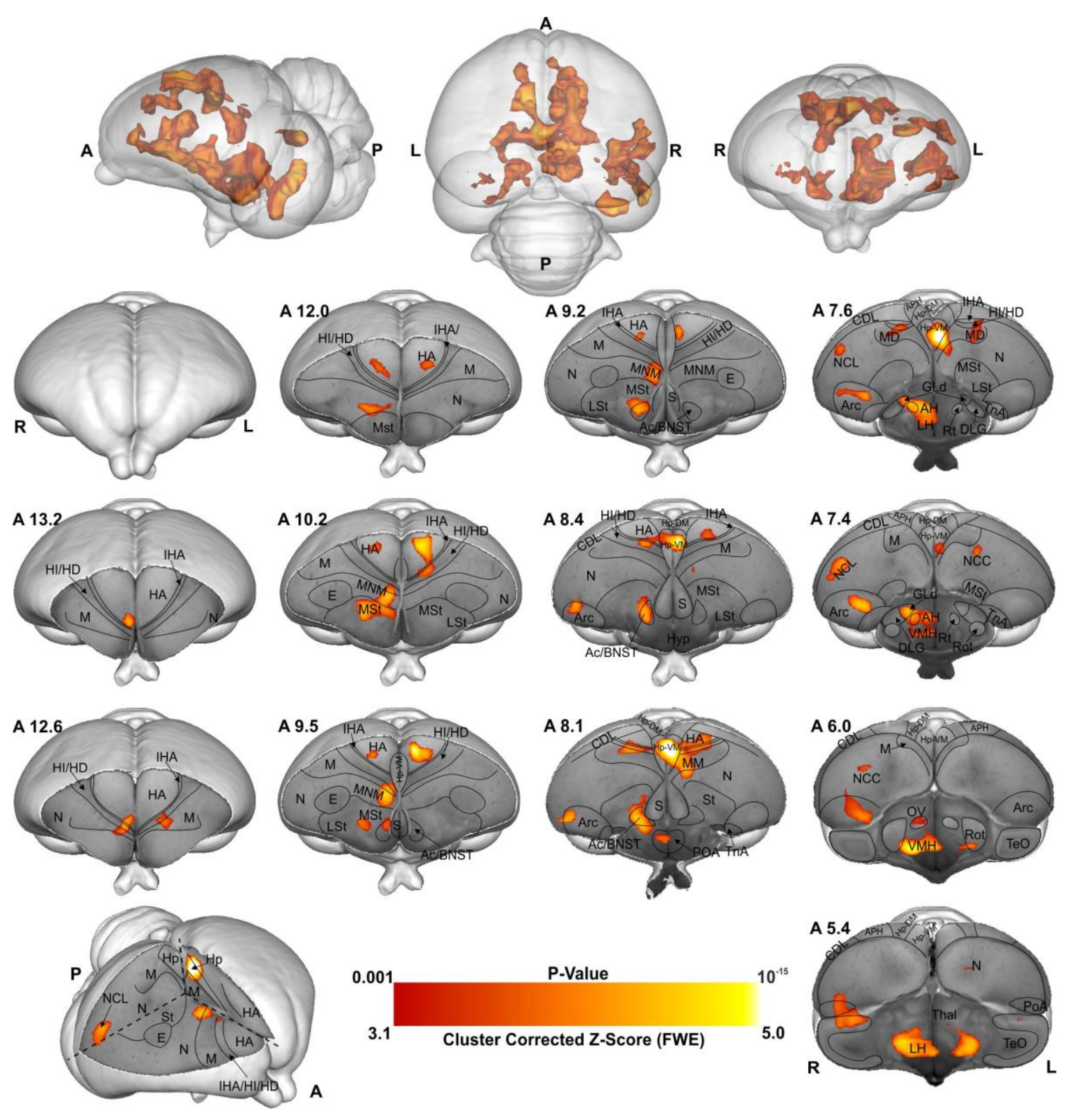

224 Fig.4- BOLD response pattern during imprinting memory retrieval. The high-resolution coronal 225 slices at the different levels of an ex-vivo chick brain are in greyscale, while the contrast map 226 represents the activation pattern during the presentation of the preferred imprinting object after 227 imprinting learning has already occurred (Red group, the contrast of red light versus blue light 228 conditions, $n=9, Z=3.1$ and $p<0.05 \mathrm{FEW}$ corrected at the cluster level). The top row images 229 show the 3D representation of the activation pattern inside a translucent chick brain. A 3D 230 depiction of the chick brain is represented bottom left with an example window at the level of $A$ 231 7.4. Anatomical borders (black lines) are based on the contrast difference of ex-vivo chick brain 232 and Chick atlas (Herold et al., 2014; Kuenzel and Masson, 1988). The corresponding 233 abbreviations of ROIs are listed in the Table S1. 


\section{Discussion}

Imprinting, a well-known form of early learning, has been widely used in the 70's as

237 a model to study the neurobiology of memory formation (reviews in Horn, 2004; Rose,

238 2000). Evidence for a crucial role played by the intermediate medial mesopallium (IMM)

239 and NMm (jointly labelled as MNM) during the acquisition of imprinting memory was

240 obtained. Further studies showed that the store in the IMM is only temporary, and that a

241 transfer of information to another, unknown brain region, dubbed S' (Honey et al., 1995),

242 occurs after approximately 6 hours. These studies were conducted with either

243 autoradiographic or lesion techniques and were unable to discover the full network of

244 imprinting (Horn et al., 1979). Here we established a new fMRI protocol to study awake

245 brain activity in newly hatched domestic chicks in order to discover the neural pathways

246 of imprinting and the location of S'. After two days of imprinting training, with either a

247 preferred (red) or a non-preferred (blue) colour (Maekawa et al., 2006), chicks were

248 exposed to a sequence of the two stimulus colours inside the scanner. We provided

249 evidence for a network of brain regions involved in the acquisition of a secondary

250 imprinting memory (blue stimulus), and, in parallel, a neural network involved in the long-

251 term storage of imprinting memory (red stimulus).

252 Visual information reaches the pallium both via the tecto- and the thalamofugal visual

253 pathways. We observed a partial involvement of the nucleus rotundus (Rot), the thalamic

254 link of the tectofugal pathway during acquisition (Fig. 5A). A rotundal involvement had

255 already been reported in imprinted chicks (Harvey et al., 1998) and together with the

256 present results, it suggests a minor tectofugal role during the early stages of imprinting

257 learning. In contrast, the thalamofugal visual system seems to play a crucial role in 
258 processing imprinting information (as also reviewed in Nakamori et al., 2013). This 259 pathway consists of the retinorecipient GLd (Aoki et al., 2015) that projects to the the 260 interstitial nucleus of the hyperpallium apicale (IHA) of the visual Wulst, from where 261 secondary projections reach the three pseudo-layers of the wulst hyperpallium 262 densocellulare (HD), hyperpallium intercalatum $(H I)$, and hyperpallium apicale $(H A)$ 263 (Stacho et al., 2020). We discovered both during memory formation and retrieval (Fig. 264 5B) significant activity patterns of all these thalamofugal components. Indeed, HD of dark265 reared chicks exhibits topographically organised responses for red and blue objects 266 (Maekawa et al., 2006). After imprinting on either one of the two colours, such 267 organisation changes along the rostro-caudal axis showing imprinting-related plasticity 268 already in the Wulst.

269 Previous studies showed that Wulst lesions lead to anterograde amnesia of visual 270 imprinting memory (Maekawa et al., 2006). This possibly results from the loss of visual 271 projections from HD to IMM (Nakamori et al., 2010; Shimizu et al., 1995), the associative 272 medial pallial area that is crucial for the acquisition of imprinting memory (Horn, 2004). 273 IMM projects back to HA, establishing a loop (Aoki et al., 2015). IMM, the ventrally located $274 \mathrm{NMm}$ and the nidopallium caudolaterale (NCL) have been shown to be involved during 275 visual as well as auditory filial imprinting (Bock et al., 1997; Bredenkötter and Braun, 276 1997). Here we report a significant brain activation in IMM, NMm, and NCL during memory 277 retrieval and, to a much lesser extent, in IMM and NMm during memory formation. Indeed, 278 NMm and NCL undergo long-lasting synaptic changes after multimodal (visuo-auditory) 279 imprinting training (Bock et al., 1997; Horn, 2004; Metzger et al., 1998). Imprinting training 280 also impacts cell proliferation in NMm and NCL, but not in IMM (Komissarova and 
281 Anokhin, 2008). Thus, these three areas play important but differential roles in multimodal

282 filial learning and the subsequent formation of long-term memory. Note that in the present

283 study chicks were also exposed to the noise produced by the scanner. Thus, NMm and

$284 \mathrm{NCL}$ on the one and the auditory n. ovoidalis (OV) - Field-L pathway on the other side, 285 conceivably constituted the neural basis for the acoustic component of acquiring (blue 286 group) or retrieving imprinting memory (red group).
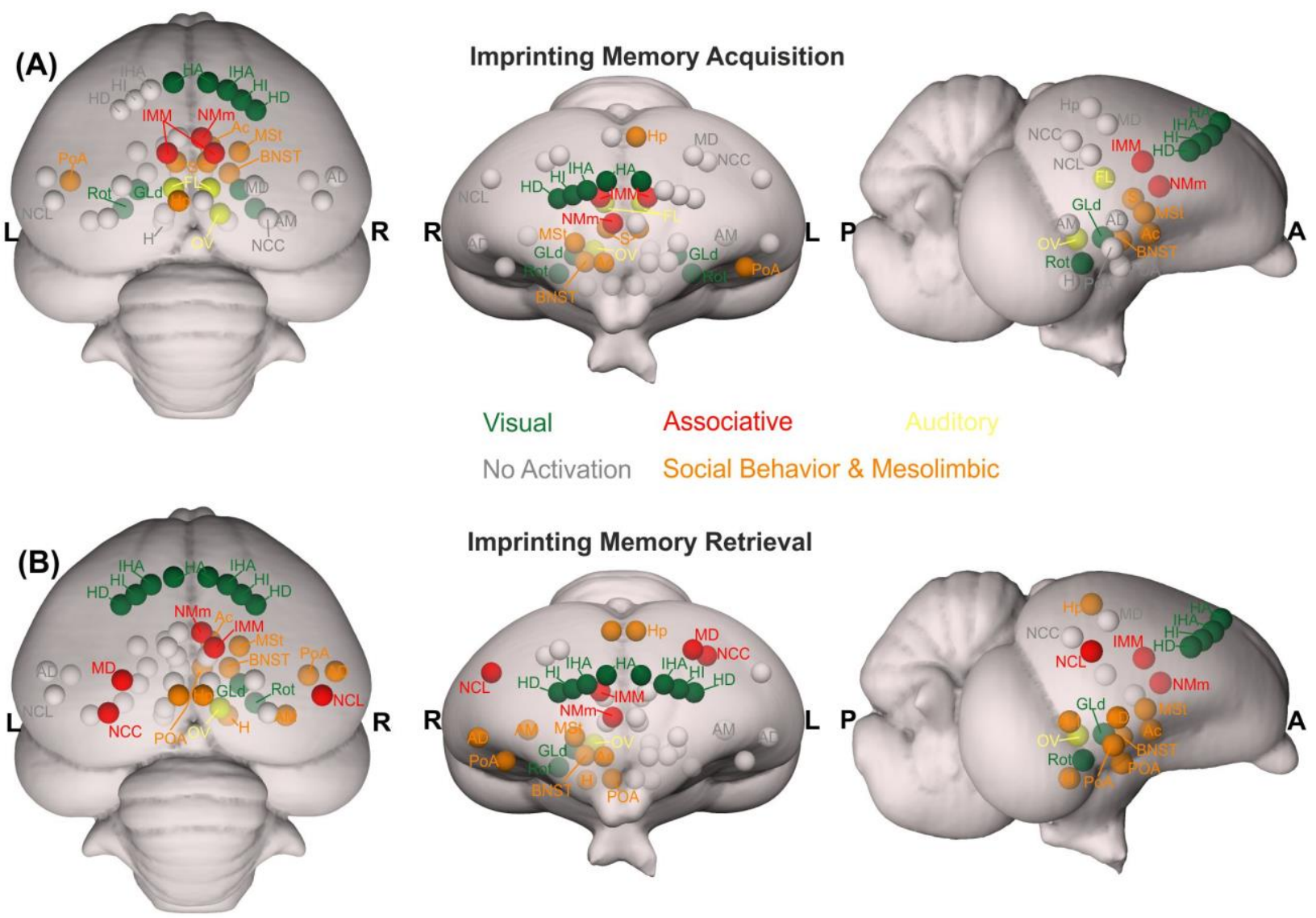

288 Fig.5- Schematic depiction of the activated prosencephalic areas during different phases of 289 imprinting memory. (A) Network activated during imprinting memory acquisition are represented in colourful circles. (B) Network activated during imprinting memory retrieval are represented in 291 colourful circles. The grey circles represent no activation. The corresponding abbreviations of 292 ROIs are listed in the Table S1. 
But the interconnected higher associative regions, NMm and NCL do not only play 295 a role for long-term memory-related mechanisms (Atoji and Wild, 2012; Behroozi et al., 296 2020; Metzger et al., 1998; Rook et al., 2021). NMm is also involved in sensorimotor 297 learning and sequential behaviour (Helduser and Güntürkün, 2012), while NCL, largely 298 accepted as a prefrontal-like field (von Eugen et al., 2020), is involved in working memory 299 (Diekamp et al., 2002; Veit et al., 2014), executive control (Rose and Colombo, 2005) and 300 in merging multi-sensory information in long-term memory engrams (Moll and Nieder, 301 2015). This evidence together with the present findings further supports the involvement 302 of these regions in the long-term storage and flexible retrieval of a multimodal imprinting 303 memory trace.

304 The motor output component of NMm and NCL is established by their projections to 305 arcopallium and medial striatum (MSt, (Atoji and Wild, 2012; Csillag, 1999; Kröner and 306 Güntürkün, 1999; Metzger et al., 2002; Schnabel et al., 1997)). Possibly, the initially 307 pallially processed imprinting trace is thereby transferred into a striatum-dependent 308 response strategy. As a result, striatal S-R associations are formed and once acquired, 309 dominate the behaviour of the animal (Csillag, 1999). This also has been shown for 310 passive avoidance learning. Here, the mnemonic nature of MSt (previously lobus 311 paraolfactorius (Reiner et al., 2004)) goes hand in hand with that of IMM (Gibbs, 2008; 312 Rose, 2000; Sojka et al., 1995), with increased density of synapses and dendritic spines 313 being detectable some days after training in MSt, but not in IMM (Bradley et al., 1985; 314 Sojka et al., 1995; Stewart and Rusakov, 1995). Additionally, after imprinting training 315 glutamate receptor binding affinity increases both in MSt and arcopallium (Izawa et al., 
316 2001; Johnston et al., 1993; McCabe and Horn, 1994), while, pre-imprinting arcopallial

317 lesions impair memory acquisition (Lowndes et al., 1994).

318 We found enhanced brain activity in the most medial part of MSt both during

319 acquisition and retrieval of imprinting memory, while for the dorsal and medial portions of

320 arcopallium this was only observed for retrieval. These portions of MSt and arcopallium

321 are enriched in the limbic system-associated membrane protein (LAMP, (Yamamoto and

322 Reiner, 2005)). We also found a strong mesolimbic involvement in imprinting memory in

323 the two interconnected Social Behavior Network and Mesolimbic Reward System

324 (Goodson and Kingsbury, 2013; Newman, 1999; O'Connell and Hofmann, 2011). Here

325 septum was involved only during memory formation. Arcopallium, preoptic area, anterior

326 and ventromedial hypothalamus (POA, $\mathrm{AH}, \mathrm{VMH})$ were involved only during memory

327 retrieval. In contrast, Hp, MSt, bed n. of the stria terminalis (BNST), n. accumbens (Ac)

328 and posterior pallial amygdala ( $P \circ A)$ were involved during both memory formation and

329 retrieval. While involvement of these systems in social predispositions associated with

330 imprinting had already been observed (Lorenzi et al., 2017; Lorenzi and Vallortigara,

331 2021; Mayer et al., 2017), this is the first evidence for their involvement during imprinting.

332 Such involvement could represent the motivational component linked to the association.

333 Indeed, in the context of filial imprinting, emotional-motivational engagement must be

334 particularly pronounced at different stages of the learning process. The septum seems to

335 be preferentially involved during the first stages of imprinting and probably driving the

336 chick's attention toward salient predisposed moving stimuli. Previous studies also

337 revealed septal involvement during the first exposure to a red object moving with abrupt

338 changes of speed or an alive conspecific (Lorenzi et al., 2017; Mayer et al., 2017). 
339 Although BNST, Ac, MSt, and PoA seem to participate in both imprinting memory

340 formation and retrieval, we found greater activity in the red group. Such enhanced activity

341 may suggest a stronger emotional-motivational component after memory consolidation of

342 the imprinting engram.

343 The HD of the Wulst has bidirectional connections with PoA and Hp (Atoji et al.,

$3442018,2006)$. We found a hippocampal (Hp) involvement both during imprinting memory

345 formation and retrieval. The hippocampal formation is known for its role in memory in

346 birds and mammals (Colombo and Broadbent, 2000). However, $c$-fos immunoreactivity in

347 chicks revealed also a social role of $\mathrm{Hp}$. The dorso- and ventromedial portions are

348 involved in individual recognition in chicks (Corrales Parada et al., 2021). The same

349 portions here were found to be involved in imprinting memory, strengthening a regional

350 specialisation of hippocampus dedicated to social memory functions. Indeed, Hp projects

351 ipsi- and contralaterally to IMM (Bradley et al., 1985) and is involved in filial imprinting

352 (McCabe and Horn, 1994). We found a left Hp involvement during filial imprinting memory

353 formation (blue group), and a bilateral one during memory retrieval (red group).

$354 \quad$ Interestingly, the brain activity pattern was predominantly right lateralised. Among

355 the exceptions was a left $\mathrm{Hp}$ involvement during imprinting memory formation (blue

356 group), and a bilateral $\mathrm{Hp}$ involvement during memory retrieval (red group). Lateralisation

357 is a common feature in the avian brain, especially at different stages of memory formation

358 (Moorman and Nicol, 2015; Rogers et al., 2013; Tulving et al., 1994). Right lateralisation

359 during memory formation has been reported for passive avoidance learning (Lössner and

360 Rose, 1983). Instead, for imprinting learning, time-shifts have been observed in the

361 lateralisation pattern of IMM. The left IMM is involved at first in learning the features of 
362 the imprinting object, while the right IMM dominates during memory consolidation and the

363 subsequent establishment of the long-term storage S' (Cipolla-Neto et al., 1982; McCabe,

364 1991). A similar pattern of lateralisation has been proposed in the hemispheric

365 encoding/retrieval asymmetry model (HERA) in humans, where the left hemisphere plays

366 a dominant role during memory encoding and the right during retrieval (Tulving et al.,

367 1994). Such evidence together leads to the hypothesis of a dual memory system for

368 imprinting, in which different processes - acquisition and consolidation - take place in

369 different hemispheres, with prominent right lateralisation for consolidation processes

370 (Cipolla-Neto et al., 1982). Indeed, during memory consolidation, a glutamate injection

371 into the right IMM disrupts imprinting memory, but it does not when injected into the left

372 hemisphere (Johnston and Rogers, 1998). Our results may add a novel view on the idea

373 of the dual memory system: While the visual thalamofugal nucleus GLd was bilaterally

374 activated during acquisition, only the right side was active during retrieval. It is

375 conceivable that right hemispheric memory consolidation increased top-down projections

376 onto right sided sensory thalamic nuclei in order to focus attention on learned object

377 properties (Kastner and Ungerleider, 2000). This then could activate and synchronize

378 right hemispheric pallial areas according to attentional allocation, thereby inducing a right

379 hemispheric superiority in imprinting memory retrieval (Saalmann et al., 2012).

380 Our findings could for the first time uncover a prosencephalic neural network that,

381 among others, involves the Social Behavior Network, the Mesolimbic Reward System,

382 and the medial meso-/nidopallium for long-term storage and retrieval of filial imprinting

383 memory. As to be expected, the networks involved in memory formation and retrieval

384 partially overlapped. However, network activity was more pronounced and further 
385 involved arcopallium and NCL in the retrieval condition. Thus, consolidation of imprinting 386 memory results in a strengthening and expansion of the neural system that holds the 387 engram in distributed manner. Within this perspective, the long searched site for 388 imprinting memory dubbed as S' by Gabriel Horn (Horn, 1985) is possibly this whole 389 network within which the "prefrontal" NCL could be a central hub. 


\section{Materials and methods}

\section{Subjects}

All procedures here presented followed all the applicable European Union and Italian

393 laws, and guidelines for animals' care and use and were approved by the Ethical

394 Committee of the University of Trento OPBA and by the Italian Health Ministry (permit 395 number 738/2019).

396 A local commercial hatchery (Azienda Agricola Crescenti, Brescia, Italy) provided

397 fertilised eggs of the Aviagen Ross 308 strain (Gallus gallus domesticus). Eggs were

398 incubated and hatched in the laboratory under controlled temperature $\left(37.7^{\circ} \mathrm{C}\right)$ and

399 humidity (60\%) in darkness using FIEM MG140/200 Rural LCD EVO incubators. Soon

400 after hatching, chicks were sexed by feather dimorphism, with a black cap on the head in

401 order to prevent any visual stimulation. Twenty-six females were used in the present

402 study. Females were used because they are known to exhibit stronger filial attachment

403 with the imprinting object (Cailotto et al., 1989; Vallortigara, 1992; Vallortigara et al., 404 1990). Each chick underwent the experimental procedure only once. At the end of the 405 experimental procedure, on post-hatching day 3, chicks were caged in groups with water 406 and food ad libitum, at constant temperature $\left(32.3^{\circ} \mathrm{C}\right)$ and with a $12: 12$ day-night light 407 cycle until they were donated to local farmers.

409 On the day of hatching, chicks were caged individually at a constant temperature of $41032.3^{\circ} \mathrm{C}$ with water and food. In each cage $(28 \times 40 \times 32 \mathrm{~cm})$ the imprinting stimulus, a hollow 411 plastic ball (diameter $3.5 \mathrm{~cm}$ ), was suspended in the middle $(7 \mathrm{~cm}$ from the floor, Fig. 1 
412 A). Two optical fibres (diameter of $2 \mathrm{~mm}$ ) inserted in the ball were flickering at $5 \mathrm{~Hz}$. Chicks

413 prefer to imprint on a flickering than on a stationary light (James, 1959). For one group of

414 chicks, the ball was flickering with red light $(\mathrm{N}=9$, dominant wavelength $=642 \mathrm{~nm}$,

415 intensity $\left.=16.45 \mathrm{~cd} / \mathrm{m}^{2},\right)$, for the other group with blue light $(\mathrm{N}=8$, dominant wavelength

$416=465 \mathrm{~nm}$, intensity $\left.=16.45 \mathrm{~cd} / \mathrm{m}^{2}\right)$. Being the only light provided in the environment, the

417 established setup by Behroozi et al. (Behroozi et al., 2020) and a custom-written MATLAB

418 code were used to automatically switch on and off the light, following a day-night cycle

419 12:12. During the daytime, to habituate the subjects to the noise of the scanner, a

420 recording of the sound was provided twice per day, for a total amount of 5 hours per day,

421 by two loudspeakers (Logitech) placed outside the cages.

\section{Acquisition and Pre-processing of fMRI data}

All MRI experiments were recorded using a horizontal-bore small animal MRI

424 scanner (7.0 T Bruker BioSpin, Ettlingen, Germany) equipped with a BGA-9 gradient set

$425(380 \mathrm{mT} / \mathrm{m}$, max. linear slew rate $3,420 \mathrm{~T} / \mathrm{m} / \mathrm{s})$. A $72 \mathrm{~mm}$ transmit birdcage resonator was

426 used for radio-frequency transmission. To reduce the motion artifacts resulting from body

427 parts' movements, a single-loop $20 \mathrm{~mm}$ surface coil was placed around the chicks' head

428 for signal reception.

429 Localiser. At the beginning of each scanning session, a set of scout images

430 (coronal, horizontal, and sagittal scans) were recorded as localisers to identify the position

431 and orientation of the chick's brain inside the MRI machine. The scout images were

432 acquired using a multi-slice rapid acquisition (RARE) sequence with the following

433 parameters: repetition time $(T R)=3000 \mathrm{~ms}$, effective echo time $\left(T_{\text {eff }}\right)=41.2 \mathrm{~ms}$, RARE

434 factor $=32, \quad N$ _average $=2$, acquisition matrix $=128 \times 128$, the field of view 
$435($ FoV $)=20 \times 20 \mathrm{~mm}$, spatial resolution $=0.156 \times 0.156 \mathrm{~mm}^{2}$, slice thickness $=1 \mathrm{~mm}$,

436 number of slices $=8$, slice orientation $=$ coronal $/$ horizontal $/$ sagittal, with a total scan time

437 of $18 \mathrm{~s}$. This information has been used to position 9 coronal slices in a way $\left(\sim 40^{\circ}\right.$ 438 regarding coronal direction) to cover the entire telencephalon to record the fMRI time 439 series.

$440 \quad$ fMRI (task). The blood-oxygen-level-dependent (BOLD) time series were recorded 441 using a single-shot multi-slice RARE sequence adopted from Behroozi et al. (Behroozi et 442 al., 2020, 2018) with the following parameters: TR/TE eff $=4000 / 51.04 \mathrm{~ms}$, RARE factor $=$ 44342 , acquisition matrix $=64 \times 64$, FoV $=30 \times 30 \mathrm{~mm}^{2}, 9$ coronal slices no gap between 444 slices, slice thickness $=1 \mathrm{~mm}$, slice order $=$ interleaved. Since the eyes' size is 445 comparable to brain's one, two saturation slices were manually positioned on the eyes to 446 saturate the possible eye movement artifacts, which can corrupt the BOLD signal. A total 447 of 540 volumes were recorded for each animal.

448 fMRI (Rest). Whole-brain resting-state fMRI data (200 volumes) of nine chicks were 449 recorded using a single-shot RARE sequence with the same parameter as the task fMRI 450 sequence.

451 Structural MRI. High-resolution anatomical images were acquired using a RARE 452 sequence with following parameters: $\mathrm{TR} / \mathrm{TE}$ eff $=6000 / 42.04 \mathrm{~ms}$, RARE factor $=16$, 453 N_Average $=4$, acquisition matrix $=160 \times 160$, FoV $=30 \times 30 \mathrm{~mm}^{2}$, 39 coronal slices 454 with no gap between slices, slice thickness $=0.33 \mathrm{~mm}$, total scan time $=4 \mathrm{~min}$.

455 Experimental Task. Inside the fMRI machine, chicks were presented with two 456 different stimulus types, imprinted (red/blue) and control colour (blue/red) with the same 457 wavelength and intensity as the training phase. The light stimuli were generated using the 
458 established setup by Behroozi et al. (Behroozi et al., 2020). Stimuli were presented in a 459 pseudo-random order in an ON/OFF block design experiment. The duration of ON blocks

460 was $16 \mathrm{~s}$. ON blocks were interleaved with a rest period of $24 \mathrm{~s}$ (OFF blocks, inter-trial 461 interval (ITI)). In total, 48 trials were recorded during an $\mathrm{fMRI}$ session from each animal 462 (24 trials per stimulus).

463 Apparatus. A critical issue during awake fMRI scanning of animals is motion 464 artifacts. Therefore, immobilisation of the animal's head is essential to acquire an 465 accurate $\mathrm{fMRI}$ time series. To this end, awake chicks were immobilised in a nonmagnetic 466 custom-made restrainer, composed of a beak holder, blocks of plasticine around the head 467 to immobilise it in a comfortable way, and a round RF coil on top of the head (Fig. 1B). 468 Before the head fixation, the animal's body was wrapped in paper tissue to prevent the 469 other body parts' movement (such as wings and feet) to avoid any possible motion 470 artifacts. The animal's body inside the paper tissue was tapped to the main body of the 471 restrainer using a piece of medical tape.

All BOLD time series were pre-processed using the FMRIB Software Library (FSL,

474 version 6.0.4, https://fsl.fmrib.ox.ac.uk/fsl/fslwiki), the Analysis of Functional 475 Neurolmages (AFNI, version 20.0.09 https://afni.nimh.nih.gov/), and Advanced 476 Normalization Tools (ANTs, http://stnava.github.io/ANTs/) software. We performed the 477 following pre-processing steps for each run: (i) converting dicom files to nifti format (using 478 dcm2niix function); (ii) upscaling the voxel size by a factor of 10 (using AFNI's 3 drefit); 479 (iii) discarding the first 5 volumes to ensure longitudinal magnetization reached steady 480 state; (iv) motion correction using MCFLIRT (which aligns each volume to the middle 
481 volume of each run); (v) slice time correction to account for the long whole-brain

482 acquisition time (4000 ms); interleaved acquisitions); (vi) despiking using 3dDespike

483 algorithm in AFNI; (vii) removing non-brain tissue (using BET and manual cleaning); (viii)

484 spatial smoothing with FWHM $=8 \mathrm{~mm}$ (using FSL's SUSAN, after upscaling voxel size

485 by factor of 10); (ix) global intensity normalization with grand mean $=10000$ across

486 scanning sessions for group analysis; $(x)$ high-pass temporal filtering to remove slow drifts

487 (cut-off at 100s); (xi) anatomical brain extraction (using BET function and cleaned

488 manually); (xii) registration of the functional data to the high-resolution structural images

489 using affine linear registration (FLIRT function, six degrees of freedom). For spatial

490 normalization, a population-based template was constructed using

491 antsMultivariateTemplateConstruction.sh script (ANTs). FMRIB's Nonlinear Image

492 Registration Tool (FNRIT) (Andersson et al., 2007) was used to spatially normalize the

493 single subject anatomical images to the population-based template as a standard space.

494 The head motion of animals was quantified using framewise displacement (FD) (Power

495 et al., 2014). Three animals' data were excluded due to the excessive head motion (over

$49620 \%$ of volumes were contaminated with FD $>0.2 \mathrm{~mm}$ ). For the remaining animals, the

497 detected motion outliers were modeled as confound regressors during the general linear

498 model (GLM) analysis to reduce the impact of head motion.

\section{General linear model (GLM) analysis}

500 Whole-brain statistical analysis was performed using the FEAT (FMRI Expert

501 Analysis Tool) to assess stimulus-evoked activation patterns. Single-subject GLM

502 analysis was carried out to convolve the established double-gamma avian hemodynamic

503 response function in pigeon brain by Behroozi et al. (Behroozi et al., 2020) (the closest 
504 brain in the structural organization to the chick brain) to the explanatory variables (on/off 505 stimulation). The GLM contained the following three explanatory variables (EVs) and their

506 temporal derivatives: (i) imprinting trials (red/blue); (ii) control trials (blue/red); (iii) junk

507 trials (the first 16 trials were used as habituation period to the real magnet environment).

508 In addition, six estimated head motion parameters (three translations and three rotations)

509 and outlier volumes detected based on the FD analysis were modelled as confound EVs

510 to remove the residual motion artifacts. To perform group inference, subject-level

511 parameter estimates were taken into the second-level analysis using the mixed-effect

512 model (FLAME1+2) to produce group-level estimates of each condition. FLAME 1+2

513 cluster-based approach has been used to threshold the group-level statistical maps for

514 contrasts of interest with a cluster defining voxel threshold of $p<0.001(Z>3.1)$ for red

515 group and $p<0.01(Z>2.3)$ for blue group and Family Error Wise (FEW) cluster

516 significance threshold of $p=0.05$.

$517 \quad$ Visualization

518 To visualize the results, we took advantage of the high-resolution anatomical image

519 acquired for another study. Briefly, five post-mortem chick brains were scanned using a

520 fast-low angle shot (FLASH) sequence with following parameters: TR/TE $=50 / 4 \mathrm{~ms}$,

$521 \quad$ N_average $=6$, acquisition matrix $=400 \times 400 \times 500$, voxels size $=0.05 \times 0.05 \times 0.05$

$522 \mathrm{~mm}^{3}$, total scan time $=19 \mathrm{~h} 48 \mathrm{~min}$. The population-based template was co-registered

523 nonlinearly (using FNIRT) to the high-resolution anatomical image of the chick brain. The

524 contrasts of interest, eventually, were non-linearly warped to the high-resolution

525 anatomical image. MANGO software (http://ric.uthscsa.edu/mango/mango.html, version

526 4.1) was used for 3D visualization of the activation patterns. Surf Ice software 
527 (https://www.nitrc.org/projects/surfice/, version v1.0.20201102 64bit x86-64 Windows)

528 was used for surface rendering the chick brains with overlays to illustrate activated

529 networks during imprinting acquisition and retrieval memory.

530 


\section{Acknowledgments}

\section{Funding}

534 33538), the Deutsche Forschungsgemeinschaft (DFG, German Research Foundation)

535 through grant SFB 874 (A1, B5) project number 122679504, SFB 1280 (A01, A08, and

536 F02) project number 316803389 and Gu 227/16-1. GV acknowledges grants from the

537 European Research Council under the European Union's Seventh Framework

538 Programme (FP7/2007-2013) Grant ERC-2011-ADG_20110406, Project no:

539461 295517, PREMESOR), by Fondazione Caritro Grant Bio-marker DSA [40102839],

540 and PRIN 2015. AG acknowledges funding by the European Research Council (ERC,

541 DISCONN; no. 802371), the Brain and Behavior Foundation (NARSAD Independent

542 Investigator Grant \#25861), the NIH (1R21MH116473-01A1) and the Telethon foundation

543 (GGP19177).

$544 \quad$ Author Contributions

545 Conceptualization: Onur Güntürkün and Giorgio Vallortigara.

546 Experiment design: Mehdi Behroozi, Elena Lorenzi, Onur Güntürkün and Giorgio

547 Vallortigara.

548 Data Collection: Mehdi Behroozi, Elena Lorenzi, and Sepideh Tabrik

$549 \quad$ Methodology: Mehdi Behroozi and Sepideh Tabrik

550 Resources: Alessandro Gozzi and Giorgio Vallortigara 
$551 \quad$ Writing: All authors

552 Visualization: Mehdi Behroozi, Elena Lorenzi, Onur Güntürkün , and Sepideh 553 Tabrik.

$554 \quad$ Competing interests

$555 \quad$ The authors declare no competing interests.

$556 \quad$ Data and material availability

$557 \quad \mathrm{fMRI}$ data for the chick imprinting and resting-state $\mathrm{fMRI}$ are available at (will be 558 published after publishing manuscript). FSL software (https://fsl.fmrib.ox.ac.uk/fsl/fslwiki/, 559 version 6.0.4) and MATLAB (2020b, MathWorks, USA) were used to process fMRI and

560 behavioural data, respectively. Related processing codes can be found at

561 https://github.com/mehdibehroozi/Imprinting-fMRI. All data needed to evaluate the

562 conclusions in the paper are present in the paper and/or the Supplementary Materials. 


\section{$564 \quad$ References}

565 Andersson JLR, Jenkinson M, Smith SM. 2007. Non-linear optimisation. FMRIB 566 technical report TR07JA1. $\operatorname{Pr} 16$.

567 Aoki N, Yamaguchi S, Kitajima T, Takehara A, Katagiri-Nakagawa S, Matsui R,

568 Watanabe D, Matsushima T, Homma KJ. 2015. Critical role of the neural pathway 569 from the intermediate medial mesopallium to the intermediate hyperpallium apicale 570 in filial imprinting of domestic chicks (Gallus gallus domesticus). Neuroscience

571 308:115-124. doi:10.1016/j.neuroscience.2015.09.014

572 Atoji Y, Saito S, Wild JM. 2006. Fiber connections of the compact division of the 573 posterior pallial amygdala and lateral part of the bed nucleus of the stria terminalis 574 in the pigeon (Columba livia). J Comp Neurol 499:161-182. doi:10.1002/cne.21042

575 Atoji Y, Sarkar S, Wild JM. 2018. Differential projections of the densocellular and

576 intermediate parts of the hyperpallium in the pigeon (Columba livia). J Comp Neurol

$577 \quad$ 526:146-165. doi:10.1002/cne.24328

578 Atoji Y, Wild JM. 2012. Afferent and efferent projections of the mesopallium in the 579 pigeon (Columba livia). J Comp Neurol 520:717-741. doi:10.1002/cne.22763

580 Atoji Y, Wild JM. 2009. Afferent and efferent projections of the central caudal

$581 \quad$ nidopallium in the pigeon (Columba livia). J Comp Neurol 517:350-370.

582 doi:10.1002/cne.22146

583 Behroozi M, Chwiesko C, Ströckens F, Sauvage M, Helluy X, Peterburs J, Güntürkün O. 584 2018. In vivo measurement of T1 and T2 relaxation times in awake pigeon and rat 585 brains at 7T. Magn Reson Med 79:1090-1100. doi:10.1002/mrm.26722

586 Behroozi M, Helluy X, Ströckens F, Gao M, Pusch R, Tabrik S, Tegenthoff M, Otto T, 
Axmacher N, Kumsta R, Moser D, Genc E, Güntürkün O. 2020. Event-related functional MRI of awake behaving pigeons at 7T. Nat Commun 11:1-12. doi:10.1038/s41467-020-18437-1

590 Behroozi M, Ströckens F, Helluy X, Stacho M, Güntürkün O. 2017. Functional

591 Connectivity Pattern of the Internal Hippocampal Network in Awake Pigeons: A 592 Resting-State fMRI Study. Brain Behav Evol 90:62-72. doi:10.1159/000475591

593 Boakes R, Panter D. 1985. Secondary imprinting in the domestic chick blocked by

594 previous exposure to a live hen. Anim Behav 33:353-365. doi:10.1016/S0003-

595 3472(85)80059-2

596 Bock J, Schnabel R, Braun K. 1997. Role of the dorso - caudal neostriatum in filial

597 imprinting of the domestic chick: A pharmacological and autoradiographical 598 approach focused on the involvement of NMDA-receptors. Eur J Neurosci 9:1262$599 \quad$ 1272. doi:10.1111/j.1460-9568.1997.tb01481.x

600 Bradley P, Davies DC, Horn G. 1985. Connections of the hyperstriatum ventrale of the 601 domestic chick (Gallus domesticus). J Anat 140:577-589.

602 Bradley P, Horn G, Bateson P. 1981. Imprinting - An electron microscopic study of chick 603 hyperstriatum ventrale. Exp Brain Res 41:115-120. doi:10.1007/BF00236600

604 Bredenkötter M, Braun K. 2000. Development of Neuronal Responsiveness in the 605 Mediorostral Neostriatum/Hyperstriatum Ventrale during Auditory Filial Imprinting in 606 Domestic Chicks. Neurobiol Learn Mem 73:114-126.

607 doi:https://doi.org/10.1006/nlme.1999.3923

608 Bredenkötter M, Braun K. 1997. Changes of neuronal responsiveness in the 609 mediorostral neo striatum/hyperstriatum after auditory filial imprinting in the 

domestic chick. Neuroscience 76:355-365. doi:10.1016/S0306-4522(96)00381-8

611 Cailotto M, Vallortigara G, Zanforlin M. 1989. Sex differences in the response to social 612 stimuli in young chicks. Ethol Ecol Evol 1:323-327.

613 doi:10.1080/08927014.1989.9525502

614 Cipolla-Neto J, Horn G, McCabe BJ. 1982. Hemispheric asymmetry and imprinting: The

615 effect of sequential lesions to the hyperstriatum ventrale. Exp Brain Res 48:22-27. 616 doi:10.1007/BF00239569

617 Colombo M, Broadbent N. 2000. Is the avian hippocampus a functional homologue of 618 the mammalian hippocampus? Neurosci Biobehav Rev. doi:10.1016/S0149$619 \quad 7634(00) 00016-6$

620 Corrales Parada CD, Morandi-Raikova A, Rosa-Salva O, Mayer U. 2021. Neural basis 621 of unfamiliar conspecific recognition in domestic chicks (Gallus Gallus domesticus). $622 \quad$ Behav Brain Res 397:112927. doi:10.1016/j.bbr.2020.112927

623 Csillag A. 1999. Striato-telencephalic and striato-tegmental circuits: Relevance to 624 learning in domestic chicks. Behav Brain Res 98:227-236. doi:10.1016/S0166$625 \quad 4328(98) 00088-6$

626 Csillag A, Montagnese CM. 2005. Thalamotelencephalic organization in birdsBrain 627 Research Bulletin. Brain Res Bull. pp. 303-310.

628 doi:10.1016/j.brainresbull.2005.03.020

629 Davey J, McCabe B, Horn G. 1987. Mechanisms of information storage after imprinting 630 in the domestic chick. Behav Brain Res 26:209-210. doi:10.1016/0166-

$631 \quad 4328(87) 90180-x$

632 Diekamp B, Kalt T, Güntürkün O. 2002. Working memory neurons in pigeons. $J$ 
Neurosci 22:RC210-RC210. doi:10.1523/jneurosci.22-04-j0002.2002

634 Gibbs ME. 2008. Memory systems in the chick: Regional and temporal control by

635 noradrenaline. Brain Res Bull. doi:10.1016/j.brainresbull.2008.02.021

636 Goodson JL, Kingsbury MA. 2013. What's in a name? Considerations of homologies

637 and nomenclature for vertebrate social behavior networks. Horm Behav.

638 doi:10.1016/j.yhbeh.2013.05.006

639 Güntürkün O, Karten HJ. 1991. An immunocytochemical analysis of the lateral

640 geniculate complex in the pigeon (Columba livia). J Comp Neurol 314:721-749.

641 doi:10.1002/cne.903140407

642 Harvey RJ, McCabe BJ, Solomonia RO, Horn G, Darlison MG. 1998. Expression of the

643 GABAA receptor $\mathrm{y}$ 4-subunit gene: Anatomical distribution of the corresponding

$644 \quad m R N A$ in the domestic chick forebrain and the effect of imprinting training. Eur $J$

$645 \quad$ Neurosci 10:3024-3028. doi:10.1111/j.1460-9568.1998.00354.x

646 Helduser S, Güntürkün O. 2012. Neural substrates for serial reaction time tasks in

647 pigeons. Behav Brain Res 230:132-143. doi:10.1016/j.bbr.2012.02.013

648 Herold C, Bingman VP, Ströckens F, Letzner S, Sauvage M, Palomero-Gallagher N,

649 Zilles K, Güntürkün O. 2014. Distribution of neurotransmitter receptors and zinc in

650 the pigeon (Columba livia) hippocampal formation: A basis for further comparison

651 with the mammalian hippocampus. J Comp Neurol 522:2553-2575.

652 doi:10.1002/CNE.23549

653 Honey RC, Horn G, Bateson P, Walpole M. 1995. Functionally Distinct Memories for

654 Imprinting Stimuli: Behavioral and Neural Dissociations. Behav Neurosci 109:689_

$655 \quad 698$. doi:10.1037/0735-7044.109.4.689 
656 Horn G. 2004. Pathways of the past: The imprint of memory. Nat Rev Neurosci.

657 doi:10.1038/nrn1324

658 Horn G. 1985. Memory, imprinting, and the brain : an inquiry into mechanisms 10:315.

659 Horn G, Bradley P, McCabe BJ. 1985. Changes in the structure of synapses associated 660 with learning. J Neurosci 5:3161-3168. doi:10.1523/jneurosci.05-12-03161.1985

661 Horn G, McCabe BJ, Bateson PPG. 1979. An autoradiographic study of the chick brain

662 after imprinting. Brain Res 168:361-373. doi:10.1016/0006-8993(79)90176-8

663 Horn G, McCabe BJ, Cipolla-Neto J. 1983. Imprinting in the domestic chick: The role of 664 each side of the hyperstriatum ventrale in acquisition and retention. Exp Brain Res $665 \quad 53: 91-98$. doi:10.1007/BF00239401

666 Izawa El, Yanagihara S, Atsumi T, Matsushima T. 2001. The role of basal ganglia in 667 reinforcement learning and imprinting in domestic chicks. Neuroreport 12:1743$668 \quad$ 1747. doi:10.1097/00001756-200106130-00045

669 James H. 1959. Flicker: An unconditioned stimulus for imprinting. Can J Psychol Can $670 \quad$ Psychol 13:59-67. doi:10.1037/h0083767

671 Jarvis E, Güntürkün O, Bruce L, Csillag A, Karten H, Kuenzel W, Medina L, Paxinos G, 672 Perkel DJ, Shimizu T, Striedter G, Martin Wild J, Ball GF, Dugas-Ford J, Durand 673 SE, Hough GE, Husband S, Kubikova L, Lee DW, Mello C V., Powers A, Siang C, 674 Smulders T V., Wada K, White SA, Yamamoto K, Yu J, Reiner A, Butler AB. 2005. 675 Avian brains and a new understanding of vertebrate brain evolution. Nat Rev $676 \quad$ Neurosci. doi:10.1038/nrn1606

677 Johnston AN, Rogers LJ, Johnston GAR. 1993. Glutamate and imprinting memory: the 678 role of glutamate receptors in the encoding of imprinting memory. Behav Brain Res 
54:137-143. doi:10.1016/0166-4328(93)90072-X

680 Johnston ANB, Rogers LJ. 1998. Right hemisphere involvement in imprinting memory

681 revealed by glutamate treatment. Pharmacol Biochem Behav 60:863-871.

682 doi:10.1016/S0091-3057(98)00073-2

683 Karten HJ. 1967. The organization of the ascending auditory pathway in the pigeon

684 (Columba livia) I. Diencephalic projections of the inferior colliculus (nucleus

685 mesencephali lateralis, pars dorsalis). Brain Res 6:409-427. doi:10.1016/0006-

$686 \quad 8993(67) 90055-8$

687 Kastner S, Ungerleider LG. 2000. Mechanisms of visual attention in the human cortex.

$688 \quad$ Annu Rev Neurosci. doi:10.1146/annurev.neuro.23.1.315

689 Komissarova N V., Anokhin K V. 2008. Effects of an imprinting procedure on cell

690 proliferation in the chick brain. Neurosci Behav Physiol 38:289-296.

691 doi:10.1007/s11055-008-0041-z

692 Kröner S, Güntürkün O. 1999. Afferent and efferent connections of the caudolateral

693 neostriatum in the pigeon (Columba uvia): A retro- and anterograde pathway

694 tracing study. J Comp Neurol 407:228-260. doi:10.1002/(SICI)1096-

$69599861(19990503) 407: 2<228:: A I D-C N E 6>3.0 . C O ; 2-2$

696 Kuenzel WJ, Masson M. 1988. A stereotaxic atlas of the brain of the chick (Gallus

697 domesticus). Johns Hopkins University Press.

698 Lemche E. 2020. Research evidence from studies on filial imprinting, attachment, and

699 early life stress: a new route for scientific integration. Acta Ethol.

$700 \quad$ doi:10.1007/s10211-020-00346-7

701 Lorenz K. 1935. Der Kumpan in der Umwelt des Vogels. J für Ornithol 1935832 
83:137-213. doi:10.1007/BF01905355

703 Lorenzi E, Mayer U, Rosa-Salva O, Vallortigara G. 2017. Dynamic features of animate

704 motion activate septal and preoptic areas in visually naïve chicks (Gallus gallus).

$705 \quad$ Neuroscience 354:54-68. doi:10.1016/j.neuroscience.2017.04.022

706 Lorenzi E, Vallortigara G. 2021. Evolutionary and neural bases of the sense of animacy

707 In: Kaufman AB, Call J, Kaufman JC, editors. The Cambridge Handbook of Animal

708 Cognition. Cambridge University Press.

709 Lössner B, Rose SPR. 1983. Passive Avoidance Training Increases Fucokinase Activity

710 in Right Forebrain Base of Day-Old Chicks. J Neurochem 41:1357-1363.

711 doi:10.1111/j.1471-4159.1983.tb00833.x

712 Lowndes M, Davies DC, Johnson MH. 1994. Archistriatal Lesions Impair the Acquisition

713 of Filial Preferences During Imprinting in the Domestic Chick. Eur J Neurosci

$714 \quad 6: 1143-1148$. doi:10.1111/j.1460-9568.1994.tb00612.x

715 Maekawa F, Komine O, Sato K, Kanamatsu T, Uchimura M, Tanaka K, Ohki-Hamazaki

716 H. 2006. Imprinting modulates processing of visual information in the visual wulst of

$717 \quad$ chicks. BMC Neurosci 2006 71 7:1-13. doi:10.1186/1471-2202-7-75

718 Mayer U, Rosa-Salva O, Vallortigara G. 2017. First exposure to an alive conspecific

719 activates septal and amygdaloid nuclei in visually-naïve domestic chicks (Gallus

720 gallus). Behav Brain Res 317:71-81. doi:10.1016/j.bbr.2016.09.031

721 McCabe BJ. 2013. Imprinting. Wiley Interdiscip Rev Cogn Sci 4:375-390.

722 doi:10.1002/wcs.1231

723 McCabe BJ. 1991. Hemispheric asymmetry of learning-induced changesNeural and

724 Behavioural Plasticity. Oxford University Press. pp. 262-276. 
McCabe BJ, Horn G. 1994. Learning-related changes in Fos-like immunoreactivity in the chick forebrain after imprinting. Proc Natl Acad Sci U S A 91:11417-11421. doi:10.1073/pnas.91.24.11417

Metzger M, Jiang S, Braun K. 2002. A quantitative immuno-electron microscopic study of dopamine terminals in forebrain regions of the domestic chick involved in filial

Metzger M, Jiang S, Braun K. 1998. Organization of the dorsocaudal neostriatal complex: A retrograde and anterograde tracing study in the domestic chick with special emphasis on pathways relevant to imprinting. J Comp Neurol 395:380-404.

Moll FW, Nieder A. 2015. Cross-modal associative mnemonic signals in crow endbrain neurons. Curr Biol 25:2196-2201. doi:10.1016/j.cub.2015.07.013

738 Moorman S, Nicol AU. 2015. Memory-related brain lateralisation in birds and humans. Neurosci Biobehav Rev. doi:10.1016/j.neubiorev.2014.07.006

740 Müller-Schwarze D, Müller-Schwarze C. 1971. Olfactory imprinting in a precocial 741 mammal. Nature 229:55-56. doi:10.1038/229055a0

742 Nakamori T, Maekawa F, Sato K, Tanaka K, Ohki-Hamazaki H. 2013. Neural basis of 743 imprinting behavior in chicks. Dev Growth Differ. doi:10.1111/dgd.12028

744 Nakamori T, Sato K, Atoji Y, Kanamatsu T, Tanaka K, Ohki-Hamazaki H. 2010.

745 Demonstration of a neural circuit critical for imprinting behavior in chicks. $J$ $746 \quad$ Neurosci 30:4467-4480. doi:10.1523/JNEUROSCI.3532-09.2010

747 Newman SW. 1999. The medial extended amygdala in male reproductive behavior. A 
node in the mammalian social behavior network. Ann N Y Acad Sci 877:242-257. doi:10.1111/j.1749-6632.1999.tb09271.x

O'Connell LA, Hofmann HA. 2011. The Vertebrate mesolimbic reward system and social behavior network: A comparative synthesis. J Comp Neurol.

753 Power JD, Mitra A, Laumann TO, Snyder AZ, Schlaggar BL, Petersen SE. 2014.

754 Methods to detect, characterize, and remove motion artifact in resting state fMRI.

$755 \quad$ Neuroimage 84:320-341. doi:10.1016/j.neuroimage.2013.08.048

756 Reiner A, Perkel DJ, Bruce LL, Butler AB, Csillag A, Kuenzel W, Medina L, Paxinos G,

757 Shimizu T, Striedter G, Wild M, Ball GF, Durand S, Gütürkün O, Lee DW, Mello C

758 V., Powers A, White SA, Hough G, Kubikova L, Smulders T V., Wada K, Dugas-

759 Ford J, Husband S, Yamamoto K, Yu J, Siang C, Jarvis ED. 2004. Revised

$760 \quad$ Nomenclature for Avian Telencephalon and Some Related Brainstem Nuclei. J

$761 \quad$ Comp Neurol. doi:10.1002/cne.20118

762 Rogers LJ, Vallortigara G, Andrew RJ. 2013. Divided brains: The biology and behaviour 763 of brain asymmetries. Cambridge University Press. doi:10.1017/CBO9780511793899

765 Rook N, Tuff JM, Isparta S, Masseck OA, Herlitze S, Güntürkün O, Pusch R. 2021.

766 AAV1 is the optimal viral vector for optogenetic experiments in pigeons (Columba

767 livia). Commun Biol 4:1-16. doi:10.1038/s42003-020-01595-9

768 Rose J, Colombo M. 2005. Neural correlates of executive control in the avian brain.

$769 \quad$ PLoS Bio/ 3:1139-1146. doi:10.1371/journal.pbio.0030190

770 Rose SPR. 2000. God's organism? The chick as a model system for memory studies. 
772 Saalmann YB, Pinsk MA, Wang L, Li X, Kastner S. 2012. The pulvinar regulates

773 information transmission between cortical areas based on attention demands.

774 Science (80- ) 337:753-756. doi:10.1126/science.1223082

775 Salzen EA, Lily RE, McKeown JR. 1971. Colour preference and imprinting in domestic

776 chicks. Anim Behav 19:542-547. doi:10.1016/S0003-3472(71)80109-4

777 Schnabel R, Metzger M, Jiang S, Hemmings HC, Greengard P, Braun K. 1997.

778 Localization of dopamine D1 receptors and dopaminoceptive neurons in the chick

779 forebrain. J Comp Neurol 388:146-168. doi:10.1002/(SICI)1096-

780

781 Shanahan M, Bingman VP, Shimizu T, Wild M, Güntürkün O. 2013. Large-scale

782

783 network organisation in the avian forebrain: A connectivity matrix and theoretical

784 Shimizu T, Cox K, Karten HJ. 1995. Intratelencephalic projections of the visual wulst in pigeons (Columba livia). J Comp Neuro/ 359:551-572. doi:10.1002/cne.903590404

786 Sojka M, Davies HA, Harrison E, Stewart MG. 1995. Long-term increases in synaptic density in chick CNS after passive avoidance training are blocked by an inhibitor of protein synthesis. Brain Res 684:209-214. doi:10.1016/0006-8993(95)00403-D

791 Stacho M, Herold C, Rook N, Wagner H, Axer M, Amunts K, Güntürkün O. 2020. A

792 cortex-like canonical circuit in the avian forebrain. Science (80- ) 369.

793 doi:10.1126/science.abc5534 
794 Stewart MG, Rusakov DA. 1995. Morphological changes associated with stages of 795 memory formation in the chick following passive avoidance training. Behav Brain 796 Res 66:21-28. doi:10.1016/0166-4328(94)00119-Z

797 Tulving E, Kapur S, Craik FIM, Moscovitch M, Houle S. 1994. Hemispheric 798 encoding/retrieval asymmetry in episodic memory: Positron emission tomography 799 findings. Proc Natl Acad Sci U S A 91:2016-2020. doi:10.1073/pnas.91.6.2016 800 Vallortigara G. 2021. Born Knowing: Imprinting and the Origins of Knowledge. The MIT $801 \quad$ Press. doi:10.7551/mitpress/14091.001.0001

802 Vallortigara G. 1992. Affiliation and aggression as related to gender in domestic chicks 803 (Gallus gallus). J Comp Psychol 106:53-57. doi:10.1037/0735-7036.106.1.53

804 Vallortigara G, Cailotto M, Zanforlin M. 1990. Sex differences in social reinstatement 805 motivation of the domestic chick (Gallus gallus) revealed by runway tests with 806 social and nonsocial reinforcement. J Comp Psychol 104:361-367.

$807 \quad$ doi:10.1037/0735-7036.104.4.361

808 Veit L, Hartmann K, Nieder A. 2014. Neuronal correlates of visual working memory in 809 the corvid endbrain. J Neurosci 34:7778-7786. doi:10.1523/JNEUROSCI.0612$810 \quad 14.2014$

811 Vicedo M. 2013. The Nature and Nurture of Love. University of Chicago Press. 812 doi:10.7208/chicago/9780226020693.001.0001

813 von Eugen K, Tabrik S, Güntürkün O, Ströckens F. 2020. A comparative analysis of the 814 dopaminergic innervation of the executive caudal nidopallium in pigeon, chicken, 815 zebra finch, and carrion crow. J Comp Neurol cne.24878. doi:10.1002/cne.24878 816 Yamamoto K, Reiner A. 2005. Distribution of the limbic system-associated membrane 
817 protein (LAMP) in pigeon forebrain and midbrain. J Comp Neurol 486:221-242.

818 doi:10.1002/cne.20562

819 Yamamoto K, Sun Z, Hong BW, Reiner A. 2005. Subpallial amygdala and nucleus

820 taeniae in birds resemble extended amygdala and medial amygdala in mammals in

821 their expression of markers of regional identity. Brain Res Bull 66:341-347.

822 doi:10.1016/j.brainresbull.2005.02.016

823 


\section{chicks}

827 Mehdi Behroozi ${ }^{1,5^{*}}$, Elena Lorenzi ${ }^{2,5^{*}}$, Sepideh Tabrik ${ }^{3}$, Martin Tegenthoff ${ }^{3}$, Alessandro Gozzi ${ }^{4}$, Onur

828 Güntürkün ${ }^{1}$, Giorgio Vallortigara²

829 Corresponding authors: mehdi.behroozi@ruhr-uni-bochum.de;

830 elena.Iorenzi@unitn.it;

831 
832 Table S1. Alphabetised list of abbreviations

\begin{tabular}{|l|l|l|l|}
\hline Abb. & ROI name & Abb. & ROI name \\
\hline Ac & n. Accumbens & LH & Lateral Hypothalamus \\
\hline AD & Dorsal Arcopallium & LSt & Lateral Striatum \\
\hline AH & Anterior Hypothalamus & M & Mesopallium \\
\hline AM & Medial Arcopallium & MD & Mesopallium Dorsale \\
\hline APH & Area parahippocampalis & MM & Medial Mesopallium \\
\hline Arc & Arcopallium & NMm & nidopallium medial pars medialis \\
\hline BNST & Bed nucleus of the stria terminalis & MNM & IMM + MNm \\
\hline BOLD & Blood-Oxygen-Level-Dependent & MSt & Medial Striatum \\
\hline CDL & Area Corticoidea dorsolateralis & N & Nidopallium \\
\hline DLG & Dorsolateral Geniculate n. & NCL & Nidopallium Caudolaterale \\
\hline FL & Field-L & NCC & Central Caudal Nidopallium \\
\hline fMRI & functional Magnetic Resonance & OV & n. ovoidalis \\
\hline E & Entopallium & POA & Preoptic Area \\
\hline GLd & n. Geniculatus lateralis pars dorsalis & PoA & n. posterior pallial amygdala \\
\hline HA & Hyperpallium Apicale & RARE & rapid acquisition with relaxation \\
\hline HD & Hyperpallium Densocellulare & Rot & n. Rotundus \\
\hline HI & Hyperpallium Intercalatum & Rt & Reticular \\
\hline Hp & Hippocampus & S & Septum \\
\hline Hp- & Dorsomedial n. of the Hippocampus & St & Striatum \\
\hline Hp- & Ventromedial n. of Hippocampus & TeO & Optic Tectum \\
\hline Hyp/H & Hypothalamus & Thal & Thalamus \\
\hline IHA & Interstitial n. of Hyperpallium Apicale & TnA & n. Taeniae Amygdalae \\
\hline IMM & Intermediate Medial Mesopallium & VMH & Ventromedial hypothalamus \\
\hline ITI & Inter trial interval & & \\
\hline & & & \\
\hline
\end{tabular}


bioRxiv preprint doi: https://doi.org/10.1101/2022.02.12.480179; this version posted February 14, 2022. The copyright holder for this preprint (which was not certified by peer review) is the author/funder. All rights reserved. No reuse allowed without permission.

834

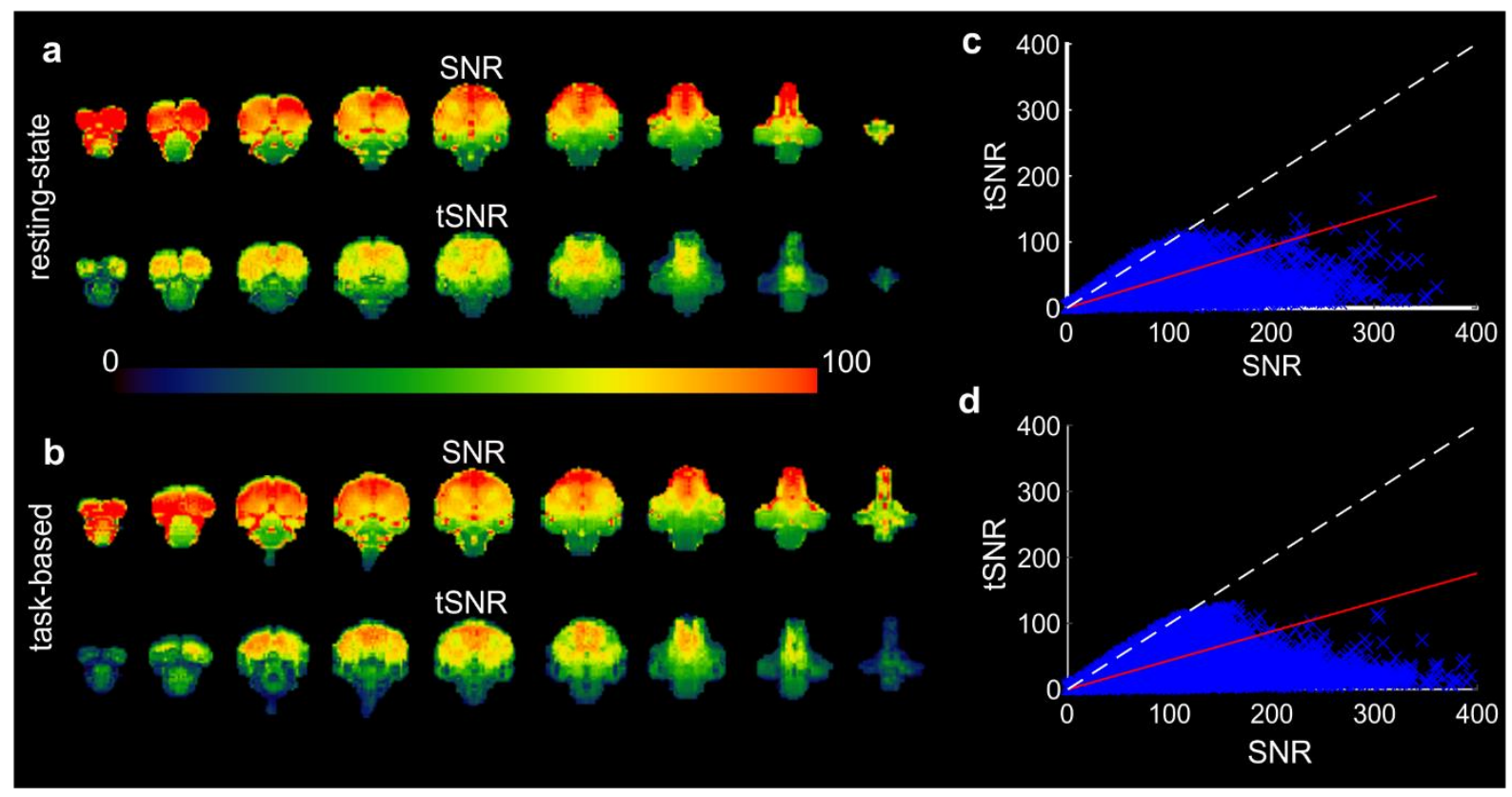

836 Figure S1- Temporal SNR and SNR assessments. (a,b) tSNR and SNR maps of resting-state and task-based fMRI 837 data. 10 min resting-state data and an imprinting functional scan were used to calculate tSNR. The voxel-wise 838 temporal SNR was defined as the voxel's temporal mean divided by its temporal standard deviation. This figure 839 illustrates that the tSNR $(>50)$ is high in the regions of interest. $(c, d)$ Overall SNR and tSNR were highly correlated 840 with each other during resting-state and task-based fMRI data. 